\title{
CONDICIONAMIENTOS DEL RÉGIMEN PARLAMENTARIO ESPAÑOL CONSECUENTES AL SISTEMA ELECTORAL ${ }^{1}$
}

\author{
LANDELINO LAVILLA ALSINA \\ Presidente de la Real Academia de Jurisprudencia y Legislación
}

La dualidad cameral de las Cortes Generales, con sus rasgos orgánicos y funcionales, es correlativa a la concepción constitucional del Congreso de los Diputados y del Senado, que, a su vez, tiene reflejo expresivo en las características del sistema electoral de acceso a uno y otro. El elemento diferencial básico de esas características es consecuente con determinaciones constitucionales, cuales son las de que el número total de Diputados se distribuirá asegurando una representación mínima inicial a cada circunscripción y asignando los demás escaños en proporción a la población (artículo 68.2) y la de que en cada provincia se elegirán cuatro Senadores (artículo 69.2). Hechas, como la Constitución las hace, las salvedades pertinentes respecto de Ceuta y Melilla y, en lo que al Senado concierne, respecto de las provincias insulares, el sistema queda constitucionalmente orientado a partir del dato de que la circunscripción electoral es la provincia.

Con tales previsiones la constitución corresponde a su propia configuración del Senado como Cámara de representación territorial. La representación de todas las provincias es paritaria, con las salvedades expresadas, y, por ende, sin atender a su población. El propio sentido de las salvedades acredita la voluntad de que unidades territoriales de acusada singularidad, ajenas (Ceuta y Melilla) o no a la división provincial, puedan tener una representación en el Senado coherente con dicha singularidad.

La realidad ya previsible -aunque germinal en el momento constituyentede las Comunidades Autónomas llevó a incorporar un elemento que complementa la inicial representación paritaria de las provincias, en cuanto adiciona un Senador por Comunidad Autónoma y otro más por cada millón de habi-

1 Palabras pronunciadas como parte del discurso de apertura del curso académico 20082009, leído el 27 de octubre de 2008.

UNED. Teoría y Realidad Constitucional, núm. 23, 2009, pp. 225-235. 
tantes de su respectivo territorio, cuya elección, de segundo grado, se verifica por la correspondiente Asamblea legislativa de la Comunidad (artículo 69.5).

El Congreso de los Diputados, respecto del que no se hace identificación constitucional explícita y paralela a la del Senado, no es, por obligada diferenciación, una Cámara de representación territorial, en el sentido que esta nota caracteriza al Senado. Podría decirse que es una Cámara de representación "poblacional", teniendo buen cuidado de no incurrir, ni siquiera en aras de una aparente mejor expresividad, en la imprecisión conceptual de referir al Congreso la representación del pueblo español que, según se ha dicho, corresponde a las Cortes Generales formada por el Congreso de los Diputados y el Senado ( artículo 66.1). Pero es lo cierto que, al preverse la distribución de escaños en proporción a la población de cada provincia, aflora la idea de que en el Congreso el número de escaños subraya el vínculo a la "población", mientras que en el Senado atiende primariamente al "territorio".

Ni uno ni otro engarce se establecen, sin embargo, de modo lineal. La exigencia de que cada provincia tenga en el Congreso una representación mínima inicial (aunque hipotéticamente ésta se fijará en tan sólo un escaño) comporta una importante corrección en atención al elemento "territorio". Del mismo modo, la atribución a la Comunidades Autónomas de un escaño en el Senado por cada millón de habitantes implica una corrección que pondera el elemento "población".

La Constitución previene que la elección para el Congreso "se verificará en cada circunscripción atendiendo a criterios de representación proporcional" (artículo 68.3), carácter éste -proporcional- de la representación que se asegurará, en todo caso, en la elección de Senadores por la Asambleas Legislativas de las Comunidades Autónomas (artículo 69.5). La Ley Orgánica Electoral, a que se remite el artículo 69.2, establece que la elección de Senadores por sufragio universal, libre, igual, directo y secreto se inspira en criterios de escrutinio mayoritario.

Con este punto de partida pueden ya exponerse algunas reflexiones sobre el sistema electoral elaboradas desde una posición teórica capaz de explicar sus características y como tributo a la propia concepción y aún, en su caso, como concesión y hasta claudicación ante los requerimientos prácticos de la política.

a) Puede haber - pero no abundan- quienes, seducidos por un atractivo eslabonamiento lógico desde sus presupuestos más teóricos, alcancen y defiendan conclusiones solo válidas en su purismo - más que en su purezapara hipotéticos e imaginarios mundos distantes de cualquier realidad conocida. La confrontación del ideal con la realidad, incluso existiendo una efectiva voluntad reformadora de ésta, no es tarea ajena a la construcción teórica sino piedra de toque de su validez y utilidad. Lejos de cualquier tentación de interferir el raciocinio y la especulación por los apremios - nobles o prosaicos- de la praxis, me considero también distante de todo exceso disquisitivo que, alcanzando las más altas cotas de sutileza y recreo intelectual, abra 
abismos entre las concepciones teóricas y el mundo de necesidades, palpables y perentorias, sobre el que me deben aquellas proyectar su luz y orientación.

Desde este punto de vista, parece necesario recordar que, en un régimen parlamentario, la bondad del sistema electoral pende de la equilibrada conjugación de dos objetivos, no propiamente contradictorios pero sí recíprocamente condicionantes: la representatividad del resultado y el favorecimiento de razonables soluciones de Gobierno. Ni la plenitud a ultranza de aquélla, con las excesivas fragmentaciones de opciones políticas y consiguientes efectos dispersivos, justifica serias y frecuentes frustraciones de la estabilidad gubernamental ni, en aras de esa, puede aparecer contradicha una representación genuina del pluralismo político.

Por ello, los diversos sistemas electorales ofrecen un variado muestrario de fórmulas en las que principios y mecanismos correctores - a veces, incluso artificios - acreditan los términos en que, con mayor o menos fortuna, se procura servir equilibradamente a los dos objetivos enunciados. El desenvolvimiento cabal de los principios elaborados y propuestos queda así tamizado por los delicados compromisos entre ideales y necesidades que cuajan en modelos más o menos próximos a una concepción pura, pero distantes de ella.

Cabe, desde luego, abrir y seguir hasta sus últimas consecuencias un debate sobre las ventajas e inconvenientes del sistema proporcional y del mayoritario. No es preciso, empero, hacer exhibiciones de pragmatismo para anticipar la convicción de que los aspectos fecundos de un tal debate aconsejan optar por un sistema "inspirado en" o "atendiendo a" criterios de representación proporcional o de escrutinio mayoritario, más que por un sistema que, de modo rígido e inexorable, sea de uno u otro tipo son correcciones ni concesiones atemperadas a la realidad u demandadas por un sano realismo.

En el sistema electoral español, la opción se ha ejercitado a favor del primer término de la disyunción para las elecciones al Congreso de los Diputados y del segundo término para las correspondientes al Senado. Ni en aquellas es puro el sistema proporcional ni lo es en éstas el sufragio mayoritario.

La valoración relativa de los sistemas no conduce por sí a la indiferencia, aunque la aproximación puede ser tal, en una hipotética línea trazada entre los extremos polares del sistema proporcional y del sistema mayoritario, que existan segmentos en los que sea apreciable cierto grado de relatividad.

El sistema electoral para el Congreso de los Diputados es, pues, proporcional con correcciones; para el Senado es mayoritario, corregido por la aplicación del sufragio restringido.

b) La constitucionalización de los criterios electorales ha merecido severos juicios críticos, por considerar inconveniente el grado de rigidez que impone. Sin embargo, no puede negarse el valor de su eficacia dirimente en una cuestión susceptible de indefinidas controversias. Tampoco cabe minusvalorar la discreción y mesura con la que la Constitución deja amplio margen para que el legislador arbitre y decida entre múltiples modalidades que admite la formulación constitucional. 
Pero ocurre, en puridad, que es superior la rigidez asociada a la inercia de un sistema electoral establecido que la que resulta de las propias previsiones constitucionales. Si el Real Decreto-Ley de 18 de Marzo de 1977, dictado al amparo de la Ley para la Reforma Política de 4 de enero de 1977, derogó la Ley electoral de Maura de 1907, que era una Ley provisional, nada de particular tiene que la Ley electoral vigente de 1985 haya prácticamente mantenido las previsiones de aquel Decreto-Ley, a pesar de ser preconstitucional y de estar especialmente concebido — según la literal dicción de su artículo 1- para regir las primeras elecciones que habían de celebrarse tras la Ley para la Reforma Política. Muchas de sus prescripciones tenían expreso o presupuesto fundamento en la singularidad de aquellos comicios y en las necesidades y características de la transición política a la sazón en curso. El mantenimiento de tales prescripciones en 1985, con leves retoques - y hasta alguna regresión - puede responder a un certero diagnóstico sobre el asentamiento y estabilización de la democracia y de los partidos. Parece, sin embargo - luego se verá en qué medida-, que rinde fiel tributo a aquella tendencia a la estabilidad de las normas electorales; tendencia prefigurada por el hecho de que su modificación profunda solo puede producirse por el definitivo impulso de quien ha resultado vencedor bajo la vigencia de las normas de cuya modificación se trata.

En cualquier caso, justo es reconocer que las previsiones constitucionales incorporan dos elementos de efectiva -y quizá innecesaria- rigidez. El primero es la fijación de la provincia como circunscripción electoral. El segundo no es sino la secuela de la inacabada, recelosa o miope concepción del Senado, puesto que las prescripciones constitucionales respecto de la elección de los Senadores es -más aún que el desdibujado diseño de la Institución- la mayor dificultad para hacer efectiva su concepción como Cámara de representación territorial.

c) Con alguna frecuencia, intensificada al hilo de los comentarios que se vierten en torno a las sucesivas consultas electorales, se subraya el número variable de votos que, en una u otra circunscripción electoral, confieren la titularidad del escaño y se destacan, al respecto, las circunscripciones entre las que el contraste aparece más acentuado. Con un cierto sentido crítico sobre el sistema y con argumentos de dispar valor, se llega a cuestionar así la igualdad del voto, cuando, para la elección de Diputados (artículo 68.1) y de Senadores (artículo 69.2), la Constitución establece el principio de sufragio igual, además de universal, libre, secreto y directo.

Una posición crítica en tales términos formulada quizá revele un juicio desfavorable sobe el modelo electoral en su conjunto; juicio al que puede prestar soporte fundado el análisis comparativo de los resultados y a partir del que cabe postular correcciones y reformas. Pero supone incurrir en un grueso error, por transposición de planos tal vez inconsciente, polarizar aquel juicio como denuncia o prueba de que el sufragio, contra lo que prescribe la Constitución, no es igual. 
El contenido jurídico del derecho de sufragio es igual en cuanto veda prácticas de las que hay experiencias históricas (como el voto plural) y mantiene con pulcritud la relación un hombre/un voto. En ese plano se despliega con sus primarias y no desdeñables exigencias el sufragio igual. Y corresponde a un plano distinto, cual es el de los resultados, el que permite ponderar el mayor o menos peso — valor - de cada voto según sea la circunscripción en que se emita y la modalidad electoral que se establezca.

Si no se diferencian ambos planos, la igualdad solo se realizaría en la abstracción de un sistema electoral utópico — con circunscripciones iguales en extensión territorial y en población- y sin posibilidad de llevarse a la práctica y, de otra parte, la propia Constitución incurriría en una irreductible contradicción interna al establecer, por ejemplo, que en cada provincia se elegirán cuatro Senadores por sufragio igual, siendo, por tanto, el mismo el número de escaños y evidentemente distinta la población en cada provincia.

Ha de afirmarse, pues, sin reservas y de modo concluyente, que la igualdad jurídica de cada voto no entra en colisión con su desigual «utilidad". Cualquier sistema electoral quedaría descalificado si la igual utilidad del voto, en la valoración final de los resultados, se erigiera en conditio juris del voto igual; ni las más simples ni las más alambicadas técnicas electorales serían capaces de cuajar en una tal ecuación. Puede haber -y naturalmente hay- mecanismos de esterilización de votos, admitidos o queridos al servicio de la racionalización del sistema, porque una ineluctable consecuencia del sufragio libre —además de igual — es el respeto al voto de quien, por convicción o por voluntad meramente testimonial, lo esterilice al darlo a una opción política carente de posibilidades o que vea frustradas sus expectativas. No puede olvidarse que, si en la diferencia de peso/valor de cada voto opera el trazado de los distritos y las características del sistema electoral, actúa también el grado de dispersión o de concentración de votos entre los candidatos, lo que es consecuencia directa y radicalmente respetable de un sufragio libre.

Guardan paralelismo con las reflexiones hechas las que a continuación cabe hacer en relación con una trasposición, también indebida, de la proporcionalidad desde el plano en el que se distribuyen los escaños entre las distintas circunscripciones a aquel en que se asignan los escaños de cada circunscripción a tenor de los resultados electorales.

El dato de que todos o parte de los escaños se distribuyan en proporción a la población de cada provincia nada dice respecto a si la elección fase lógica sucesiva - se verifica o no por un sistema proporcional y con sujeción a una u otra de sus posibles modalidades. El cotejo de los apartados 2 y 3 del artículo 68 muestra los momentos y la virtud distinta con que en cada uno de ellos se apela a la proporcionalidad. De aquí que resulten también desorientadas las posiciones que, sin percibir aquella distinción, confunden ambos planos y, erigiendo la proporcionalidad en fetiche o exacerbada fe de converso, acumulan razones conducentes a la valoración negativa del sistema electoral por insuficiente realización de los criterios de representación proporcional. 
Sin perjuicio de lo que después se dirá, ha de dejarse establecido que, cuando el artículo 68.3 de la Constitución dispone que «la elección se verificará en cada circunscripción atendiendo a criterios de representación proporcional", ni en su letra ni es su espíritu está consagrando un sistema electoral en el que la proporcionalidad se imponga matemáticamente, sino en el que se tenga presente como criterio o línea de orientación identificativa del sistema. En todo caso, el artículo 68.3 opera a efectos de determinar, atendiendo a criterios de representación proporcional, cómo se disciernen los escaños, previamente asignados a la provincia, entre los candidatos. Aun razonando en este terreno, no es ocioso reiterar que en esta fase (y por requerimientos distintos de los propios de un sistema de representación proporcional) ya han operado dos prescripciones constitucionales (la que hace de la provincia la circunscripción electoral y la que establece cómo se distribuyen los escaños entre las circunscripciones) que determinan, por sí, efectos constitucionalmente queridos, criticados con frecuencia como imperfecciones del sistema electoral y que, sin embargo, nada tienen que ver con la concepción cardinal de éste. No es imputable, por ejemplo, al sistema electoral el hecho de que, como consecuencia necesaria de aquellas prescripciones, haya cierto grado de ductilidad al cualificar el sistema — según sus resultados y en determinadas circunscripciones- como mayoritario o como proporcional. Un sistema proporcional para que empiece a ser tal y opere con cierto rigor requiere que haya un mínimo de siete escaños a distribuir. Por debajo de ese número - y a veces también por encima - la proporcionalidad puede ser inapreciable y aparentemente frustrada, según sea el número de opciones electorales y la distribución de los votos.

Algunas consideraciones adicionales se desprenderán de las reflexiones incluidas en los apartados siguientes:

d) Establecida la provincia como circunscripción electoral y siendo obligada la atención a criterios de representación proporcional (artículo 68.2 y 3), el sistema de elección de los Diputados se instrumenta técnicamente a través de listas de candidatos propuestas por los partidos, coaliciones electorales o agrupaciones de electores. Que las listas sean cerradas (el votante no puede confeccionar la suya propia escogiendo entre quienes figuran en listas de distintas candidaturas) y bloqueadas (no puede, según sus preferencias, alterar el orden en el que figuran relacionados los candidatos en la lista votada) da lugar a frecuentes críticas, de fácil armazón argumental, para evidenciar la rigidez del sistema y el acotamiento de las opciones a disposición del votante, con las consiguientes limitaciones de la efectividad democrática y con fortalecimiento de los aparatos propios de los partidos políticos.

Ninguna resistencia debe haber para aceptar un juicio desfavorable en tales términos formulado. No debe haberla, al menos, en la perspectiva desde la que la proposición crítica se formula. El estrechamiento del margen a disposición del elector y la orientación compulsiva hacia el ejercicio simplificado de la opción electoral supone, desde luego, un freno al vitalismo y espontanei- 
dad en los que deberían florecer las mejores virtudes democráticas; supone también un fuerte dirigismo desde las organizaciones partidarias que, pasando por una drástica despersonalización del voto — no importa quiénes figuran en la lista sino la sigla bajo la que comparecen y la disciplina a que se someten-, termina con un alto grado de personalización del sistema vinculado a la figura del líder del partido y que sintoniza con el cruce de rasgos presidencialistas en la realidad de un régimen definido, sin embargo, como parlamentario.

Una receptiva actitud ante aquellas críticas no es inconciliable, empero, con alguna reflexión matizadora.

En primer lugar, parece claro y generalmente reconocido que el sistema de listas cerradas y bloqueadas, introducido por el Real Decreto-Ley de 14 de marzo de 1977 por el que se establecieron las normas electorales que habrían de regir los primeros comicios — los celebrados el 15 de junio siguiente-, resultaba a la sazón obligado — más que simplemente aconsejable o justificado- por la necesidad de operar con el deliberado propósito de que la implantación y la vertebración de las opciones políticas fueran positivamente estimuladas y no obstaculizadas ni dejadas siquiera al albur de las circunstancias en transformación. ¿Cuándo la consolidación del sistema y la evolución de las condiciones permitirán o exigirán aliviar las normas electorales de aquellas prevenciones e impulsos normativos de orientación? Las respuestas pueden ser varias. Y, desde luego, se comprueba que el legislador de 1985 no consideró llegado el momento, puesto que reiteró el planteamiento y la concepción de 1977.

En segundo lugar, forzoso es reconocer que, en unas elecciones por el sistema de listas - me refiero naturalmente a elecciones generales, pues otra cosa podría ocurrir en las de carácter local, aunque en las grandes ciudades la situación no sería demasiado distinta-, el porcentaje de votantes que llevan su reflexión responsable hasta discernir el orden en que los candidatos se hallan propuestos (lista desbloqueada) o su personalización del voto hasta confeccionar lista propia con nombres tomados de dos o más listas de candidatos (listas abiertas) es, según se halla empíricamente comprobado, suficientemente reducido como para no alterar, por lo general, los resultados que se siguen del sistema de listas cerradas y bloqueadas.

Es más. La experiencia política española de estos años revela como dato - ¿sorprendente? - que en las elecciones en que no se da el sistema de lista - elecciones al Senado-, sino que cada votante puede elegir según sus preferencias entre todos los Senadores candidatos con independencia del partido que haya presentado sus candidaturas, se acusa un marcado paralelismo de resultados con los que se siguen de la elección al Congreso por el sistema de lista. Ha habido naturalmente diferencias, notorias en algún comicio o en algunas circunscripciones por la irrupción de candidaturas que no eran propiamente de partido (Senadores para la Democracia o Entessa dels Catalans, en las elecciones de 1977), pero difícilmente se puede afirmar una sólida y ni siquiera sensible personalización del voto a favor de unos u otros 
Senadores. Lo normal es que en las circunscripciones en que un partido obtiene la mayor votación a favor de su lista al Congreso obtenga también tres escaños al Senado (los tres que corresponden por aplicación del sufragio mayoritario restringido). Esta tendencia ha quedado reforzada en la Ley Electoral de 1985 por una previsión cuya aparente nimiedad no disipa la percepción de su fuerza psíquica: los candidatos al Senado se agrupan en la papeleta por partidos proponentes y con las siglas de éstos, de modo que, incluso visualmente, las candidaturas parecen "como de" lista de partido. Con anterioridad y según las previsiones del Decreto-Ley de 1977, las papeletas contenían la relación de candidatos por orden alfabético aunque figurara también, junto el nombre de cada uno, el logotipo del partido que hubiera presentado la candidatura.

En tercer lugar, finalmente, no puede prescindirse de la adicional complejidad del escrutinio que, frente a la simplicidad del sistema actual, tendría el desbloqueo o apertura de las listas. Este hecho, junto al dato del escaso porcentaje de votantes que presumiblemente harían uso de las nuevas posibilidades y a la conciencia dispar acerca de si el pluralismo y la situación de las organizaciones partidarias han alcanzado o no el grado suficiente de solidez, explica quizá las dificultades, que pueden antojarse excesivas, para avanzar por el camino a que apuntan las críticas inicialmente aludidas y que, a no tardar, se verán superadas por el imparable desarrollo de las nuevas tecnologías.

e) Son varias las fórmulas que, desde la propia ciencia política o desde otras ciencias colindantes o no -incluida la matemática-, han alumbrado fórmulas y procedimientos para hacer efectiva la regla de proporcionalidad en la atribución de los escaños, según el número de votos obtenidos por cada candidatura. Como es obvio, el problema a resolver —expresivamente identificado como el de los restos- deriva de que los efectivos votos otorgados a cada lista solo casualmente coincidirían con el cociente electoral (división del número total de votos por el de escaños a repartir) o con sus múltiplos. El rigorismo conducente a la mayor aproximación posible a la proporcionalidad, con todas sus consecuencias, genera una sensible dificultad en la aplicación de algunas de aquellas fórmulas - más allá de la propia complicación del escrutinio-, a la vez que favorece, al evitar en el mayor grado posible la esterilidad del voto (incluso mediante un reparto a escala nacional de escaños atendiendo a los restos no consumidos en la adjudicación de los correspondientes a cada circunscripción), una cierta dispersión del voto y consiguiente fragmentación, que puede llegar a ser indeseable en la composición final de la Cámara.

Si se recuerdan los dos objetivos inicialmente asignados al sistema electoral y sólo alcanzables de modo relativo (la representatividad de las Cortes y el alumbramiento de soluciones de Gobierno), cabría decir que el alambicado desarrollo de las fórmulas de adjudicación de escaños, al servicio del resultado más cercano a la proporcionalidad, puede atender, en relación inversa, los requerimientos de la representatividad y las exigencias de racionalidad parla- 
mentaria y de estabilidad gubernamental. La mejor conjugación de aquellos requerimientos y estas exigencias se halla presente, con brillantez, en algunas reglas que, a través de diversos mecanismos, priman la concentración de votos en torno a una opción y, paralelamente por tanto, penalizan su excesiva dispersión.

El sistema electoral español ha seguido la regla d'Hont que no solo atiende a los dos objetivos señalados sino que lo hace mediante una mecánica operativa en la que se resuelve simultáneamente - al menos, en teoría - el problema de los restos, al verificar la adjudicación de escaños según el criterio del mayor cociente en una escala de cocientes sucesivos hasta consumir el número de escaños a adjudicar.

Es obvio que la regla d'Hont articula el reparto proporcional primando a las listas más votadas y llegando a esterilizar incluso los votos de las que menos apoyo electoral han recibido. Es tópico, en el análisis de cualquiera de los comicios, evidenciar las desviaciones, poniendo en contraste el porcentaje de votos y el de escaños obtenidos, con frecuentes y correlativas imputaciones al señor Hont o, con más propiedad, al sistema electoral que aplica la regla por él propuesta. Las desviaciones son reales; suele serlo también su magnificación y, desde luego, resulta patentemente injusta su simplificada imputación a la regla d'Hont.

Tras dejar establecido que, en términos generales y aun propiciadas las compensaciones entre circunscripciones, las desviaciones producidas en las sucesivas elecciones no legitiman - ni por el sentido de la resultante ni tan siquiera por su dimensión - una descalificación del sistema electoral, conviene hacer algunas consideraciones para detectar desde la teoría el origen de aquellas desviaciones prácticas desvelando, en consecuencia, los verdaderos extremos a que, en su caso y momento, deberían apuntar las eventuales iniciativas de corrección.

Ahora, naturalmente, la cuestión considerada es la del reparto de escaños entre las distintas listas resultando orillados, por tanto, los extremos inicialmente asociados a la vigencia de un sistema inspirado en criterios de representación proporcional (con arreglo a la Constitución) y de listas cerradas y bloqueadas (con arreglo a la Ley Electoral).

El rigor en la concepción de la regla d'Hont se despliega, en términos difícilmente controversibles, cuanto los criterios de proporcionalidad son aparentes de modo razonable y efectivo. Y lo son desde un determinado número de escaños a repartir (suele fijarse en siete, como antes he dicho), por debajo del cual no es que la regla d'Hont acuse defectos o insuficiencias, sino que en el sistema pierde fuerza y gana indiferencia el principio de proporcionalidad. Cuanto mayor es el número de escaños a adjudicar mayor es la posibilidad de que el sistema proporcional sea auténticamente tal o avance en su aproximación a la plenitud. Y cuanto más puedan actuar los criterios de proporcionalidad más lucirá el rigor distributivo de la regla d'Hont. Por ello, en las elecciones al Parlamento Europeo (con colegio electoral único) o en las elecciones de Diputados por Madrid y Barcelona (con un número de escaños 
superior a treinta) apenas se advierten desviaciones o se levantan críticas salvo las fundadas en excesos pusilánimes sin verdadera relevancia práctica. Por el contrario, en las circunscripciones con un reducido número de escaños los desajustes pueden ser - son- llamativos y, al no ser pocas tales circunscripciones, dichos desajustes se proyectan sobre los resultados globales. Es erróneo, sin embargo, imputar a la regla de distribución de escaños según criterios de proporcionalidad - y esa imputación a la regla d'Hont, a sus "artes" o a sus "misterios", suele hacerse- lo que derechamente se sigue de la falta de los presupuestos para que la proporcionalidad pueda operar de modo propio. Basta considerar que, en trance de distribuir tres escaños (y a varias circunscripciones españolas no les corresponde un número superior), el sistema, sea una u otra la regla que se aplique, está más cerca del mayoritario con sufragio restringido que del proporcional.

Existe un dato que resulta especialmente revelador a este respecto. En el sistema electoral se ha introducido el criterio del mínimo estéril, por virtud del cual dejan de computarse a efectos de la adjudicación de escaños aquellas listas que no hayan obtenido el 3\% (en Alemania es el 5\%) de los votos. Pues bien, ese mínimo estéril (con sus singularidades en España al operar por referencia a las circunscripciones y no al todo, en atención originaria a la realidad de los partidos nacionalistas) solo adquiere relevancia en Madrid y Barcelona (y quizá en alguna provincia de alta población según el grado de dispersión de voto), porque en las demás el efecto esterilizador de la regla d'Hont es muy superior al que resulta de la aplicación de aquel mínimo. En la mayoría de las provincias españolas se quedan sin escaño listas que obtienen porcentajes de votación holgadamente superiores al 3\%. Pero, como se ha dicho, ello es consecuencia de las limitaciones con que en tales provincias opera la proporcionalidad y no de defectos achacables técnicamente al juego de la regla d'Hont.

En cualquier caso, cabe concluir significando que, no obstante algunas dificultades constitucionales para alumbrar una correcta construcción (dificultades que para algunos — no para mí - son obstáculos insalvables), la superación o atenuación de las desviaciones podría hacerse si un determinado número de escaños (¿quizá los 50 consecuentes a una ampliación del número de Diputados hasta 400, como la Constitución permite?) se adjudicaran atendiendo a los restos no utilizados en las asignaciones provinciales. Las fórmulas utilizables pueden ser diversas. Ciertamente, cabría pensar que se violentaría el principio constitucional que establece la provincia como distrito electoral, al operarse en esa distribución final de escaños "como si" hubiera un colegio electoral único. Entiendo que la Constitución no resultaría vulnerada y mucho menos si se subraya que, no solo no deja de ser la provincia la circunscripción electoral, sino que se articulan fórmulas de escrutinio y de asignación de escaños que tratan de intensificar la efectividad de los criterios de representación proporcional que la Constitución proclama en su artículo 68.3. 
TITLE: Conditions of the spanish parlamentary regimen as a result of the electoral system.

ABSTRACT: In this work some reflections are exposed on the eletoral system and his characteristics.

RESUMEN: En este trabajo se exponen algunas reflexiones sobre el sistema electoral $y$ sus características.

KEY WORDS: Parlamentary regimen. Electoral system.

PALABRAS ClaVE: Régimen parlamentario. Sistema electoral. 